\title{
Purslane Suppresses Osteoclast Differentiation and Bone Resorbing Activity via Inhibition of Akt/GSK3 $\beta$-c-Fos-NFATc1 Signaling in Vitro and Prevents Lipopolysaccharide-Induced Bone Loss in Vivo
}

\author{
Ju-Young Kim, ${ }^{a, \#}$ Hyun Mee Oh, ${ }^{b, \#}$ Sung Chul Kwak, ${ }^{c}$ Yoon-Hee Cheon, ${ }^{d, e}$ \\ Myeung Su Lee, ${ }^{a, f}$ Mun Chual Rho, ${ }^{*, b}$ and Jaemin $\mathrm{Oh}^{*, a, d, e}$ \\ ${ }^{a}$ Imaging Science-Based Lung and Bone Diseases Research Center, Wonkwang University; 344-2 Sinyong-dong, \\ Iksan, Jeonbuk 570-749, Republic of Korea: ${ }^{b}$ Bioindustrial Process Research Center, Bio-Materials Research \\ Institute, Korea Research Institute of Bioscience and Biotechnology; Jeongeup, Jeonbuk 580-185, Republic of

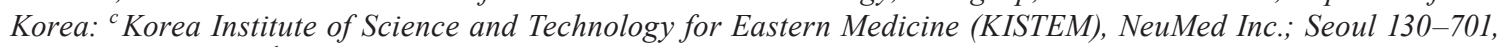 \\ Republic of Korea: ${ }^{d}$ Department of Anatomy, School of Medicine, Wonkwang University; 344-2 Sinyong-dong, Iksan, \\ Jeonbuk 570-749, Republic of Korea: ${ }^{e}$ BK21plus Program \& Department of Smart Life-Care Convergence, Graduate \\ School, Wonkwang University; 344-2 Sinyong-dong, Iksan, Jeonbuk 570-749, Republic of Korea: and ${ }^{f}$ Division of \\ Rheumatology, Department of Internal Medicine, Wonkwang University; Iksan, Jeonbuk 570-749, Republic of Korea. \\ Received August 5, 2014; accepted October 5, 2014
}

Purslane (Portulaca oleracea L.) is popular as a potherb in many areas of Europe, Asia, and the Mediterranean region and is widely distributed around the globe. It has a wide range of pharmacological effects, such as antibacterial, anti-aging, anti-inflammatory, and anti-oxidative properties. Although the extract of purslane has numerous beneficial pharmacological effects, its effect on osteoclasts remains unknown. We aimed to investigate the anti-osteoclastogenic activity in vitro and in vivo and to elucidate the underlying mechanism. The effect of purslane on the differentiation and function of bone marrow-derived macrophages (BMMs) into osteoclasts was examined using a phenotype assay such as tartrate-resistant acid phosphatase (TRAP) staining, F-actin staining, and pit assay and followed by confirmation by real-time reverse transcription polymerase chain reaction (RT-PCR) and Western blot analysis. To address the effect of purslane in vivo, the inflammatory, lipopolysaccharide (LPS)-induced osteolysis mouse model was chosen. Bone volume and bone microarchitecture were evaluated by microcomputed tomography and histologic analysis. Purslane inhibited receptor activator of nuclear factor-kappa B ligand (RANKL)-stimulated osteoclast differentiation accompanied by inhibition of Akt/glycogen synthase kinase $3 \beta$ (GSK3 $\beta$ ) signaling, which could underlie purslane-induced downregulation of c-Fos and nuclear factor of activated T cells 1 (NFATc1) expression levels, transcription factors that regulate osteoclast-specific genes, as well as osteoclast fusion and resorptionrelated molecules. Moreover, in vivo studies further verified the bone protection activity of purslane in the LPS-induced osteolysis animal model. Purslane could exhibit its anti-osteoclastogenic activity by inhibiting Akt/GSK3 $\beta$-c-Fos-NFATc1 signaling cascades. Therefore, purslane is a potential natural medicine for the treatment of osteoclast-related diseases.

Key words purslane (Portulaca oleracea); osteoclast; bone loss; osteoporosis; natural medicine

Bone is an important organ that provides mechanical support to soft tissues and maintains blood calcium and phosphate level and hematopoiesis. Both osteoblast-mediated bone formation and osteoclast-mediated resorption contribute to the dynamic remodeling process in bone tissue., ${ }^{1,2)}$ Abnormal activation of osteoclasts attributes to bone loss in many bone destructive diseases including osteoporosis, lytic bone metastases, and rheumatoid arthritis. ${ }^{1)}$ Accordingly, modulation of osteoclast differentiation and function can be a potent therapeutic target for various bone diseases characterized by excessive bone resorption.

Osteoclasts are exclusive bone-resorbing, multinucleated cells formed by the proliferation, differentiation, and fusion of hematopoietic cells belonging to the macrophage lineage., ${ }^{1,3)}$ When attached to bone matrix, multinucleated osteoclasts polarize their membrane to the bone and secrete protons and lytic enzymes such as cathepsin $\mathrm{K}$ into the resorption lacuna surrounded by a tight sealing zone. ${ }^{1)}$ The sealing zone, a char-

\footnotetext{
${ }^{\#}$ These authors contributed equally to this work.

* To whom correspondence should be addressed. e-mail: rho-m@kribb.re.kr; jmoh@wku.ac.kr
}

acteristic feature of functional osteoclasts, isolates the resorptive microenvironment from the general extracellular space. It contains ring-like structures called actin rings consisting of F-actin dots. ${ }^{4)}$

Receptor activator of nuclear factor- $\kappa \mathrm{B}$ ligand (RANKL) is the key cytokine that stimulates entire processes for the development of bone-resorbing osteoclasts. ${ }^{3,5)}$ The binding of RANKL to its receptor RANK triggers the activation of signaling molecules such as mitogen-activated protein (MAP) kinase, Akt, and nuclear factor-kappa B (NF- $\kappa \mathrm{B})$ that subsequently induce the activation of transcription factors such as c-Fos and nuclear factor of activated T cells (NFATc1) to regulate the expression of genes required for osteoclast differentiation. ${ }^{6}$ ) The proto-oncogene c-Fos is an essential factor for the induction of NFATc1, which is a master transcription factor that regulates the process of osteoclast differentiation by controlling osteoclast-specific genes. ${ }^{7-10)}$

Purslane (Portulaca oleracea L.), a member of the Portulacaceae family, is widespread as a weed in turfgrass areas as well as in field crops and has wide acceptability as a potherb in Central Europe, Asia, and the Mediterranean region. ${ }^{11)}$ 
Many studies have shown that the major bioactive components of $P$. oleracea are polysaccharides, flavonoids, coumarins, monoterpene glycoside, and alkaloids. Recent research also demonstrates that purslane has higher nutritional quality than the major cultivated vegetables, with higher levels of betacarotene, ascorbic acid, and alpha-linolenic acid. ${ }^{11,12)}$ Purslane is popular in traditional Chinese medicine and recorded in the Chinese Pharmacopoeia. It is used as a diuretic, febrifuge, antiseptic, antispasmodic, and vermifuge. ${ }^{13)}$ It has a wide range of pharmacological effects such as anti-aging, antiinflammatory, ${ }^{14)}$ antioxidative, ${ }^{15)}$ analgesic, and wound-healing activities $^{16)}$ and effects against burns, headache, and diseases related to the intestine. ${ }^{11)}$ However, a pharmacological study on its activity and mechanism in osteoclast differentiation and pathological bone destruction has not been well defined yet. In the present study, we explored the anti-osteoclastogenic effect of purslane extract in vitro and in vivo and its underlying molecular mechanism.

\section{MATERIALS AND METHODS}

Reagents Soluble, recombinant human macrophage colony-stimulating faotor (M-CSF) and human RANKL were obtained from PeproTech EC, Ltd. (London, U.K.). Lipopolysaccharide (LPS) from Escherichia (E.) coli was reconstituted in sterilized water to give a stock concentration of $10 \mathrm{mg} /$ $\mathrm{mL}$ and stored at $-20^{\circ} \mathrm{C}$ until use. Anti-p38, anti-phosphop38, anti-c-Jun N-terminal kinase (JNK), anti-phospho-JNK, anti-Akt, anti-phospho-Akt, anti-glycogen synthase kinase $3 \beta$ (GSK $3 \beta)$, anti-phospho-GSK3 $\beta$, anti-extracellular signalregulated kinase (ERK), anti-phospho-ERK, anti-inhibitor (I) $\kappa \mathrm{B}$, and anti-phospho- $\mathrm{I} \kappa \mathrm{B}$ antibodies were purchased from Cell Signaling Technology, Inc. (Beverly, MA, U.S.A.). Antic-Fos and anti-NFATc1 antibodies were purchased from Santa Cruz Biotechnology (Santa Cruz, CA, U.S.A.). A monoclonal $\beta$-actin antibody was obtained from Sigma (St. Louis, MO, U.S.A.). Fetal bovine serum (FBS), $\alpha$-minimum essential medium $(\alpha-\mathrm{MEM})$, and penicillin/streptomycin were purchased from Gibco BRL (Grand Island, NY, U.S.A.). All other chemicals were of analytical grade or complied with the standards required for cell culture.

Preparation of Purslane Extract The ethanol extract of purslane was manufactured and kindly provided by Dr. Mun Chual Rho (Bioindustrial Process Research Center, Bio-Materials Research Institute, Korea Research Institute of Bioscience and Biotechnology, Jeongeup, Korea) from the purslane harvested in Korea. Purslane was washed thoroughly with water, dried in the shade and powdered to waring brand. The powdered purslane $(12 \mathrm{~kg})$ was extracted in ethanol $(80 \mathrm{~L})$ for $7 \mathrm{~d}$ at room temperature, and the filtered extract ethanol under reduced pressure. The filtered extract was removed ethanol solvent with a vacuum rotary evaporator at room temperature, and then obtained a crude extract purslane $612 \mathrm{~g}$. Prior to the experimental use, purslane extract was dissolved in dimethyl sulfoxide (DMSO) and added to medium at a final concentration of $0.05 \%$. Serum-free medium was used as a vehicle control.

In Vitro Osteoclastogenesis Assay Bone marrow-derived cells (BMCs) were obtained from 5-week-old male ICR mice by flushing the femurs and tibias with $\alpha$-MEM supplemented with $10 \%$ FBS, penicillin $(100 \mathrm{U} / \mathrm{mL})$, and streptomycin
$(100 \mu \mathrm{g} / \mathrm{mL})$. To obtain bone marrow-derived macrophages (BMMs), BMCs were seeded on culture dishes in $\alpha$-MEM supplemented with $10 \%$ FBS and M-CSF $(10 \mathrm{ng} / \mathrm{mL})$ and cultured for $1 \mathrm{~d}$. Non-adherent cells were transferred to $10-\mathrm{cm}$ Petri dishes and further cultured in the presence of M-CSF $(30 \mathrm{ng} / \mathrm{mL})$ for $3 \mathrm{~d}$. After removal of non-adherent cells, adherent cells were used as BMMs, which are osteoclast precursors. To generate osteoclasts from these BMMs, the cells were seeded in a 48 -well plate $\left(3.5 \times 10^{4}\right.$ cells/well) in complete medium containing M-CSF $(30 \mathrm{ng} / \mathrm{mL})$ and RANKL $(100 \mathrm{ng} / \mathrm{mL})$ and cultured for $4 \mathrm{~d}$ with or without purslane. The cells were fixed in $3.7 \%$ formalin for $10 \mathrm{~min}$, permeabilized with $0.1 \%$ Triton X-100, and subsequently stained with tartrate-resistant acid phosphatase (TRAP) (Sigma). TRAP-positive multinucleated cells with more than 3 nuclei were counted as osteoclasts.

Cytotoxicity Assay The XTT (sodium 3'-[1-(phenylaminocarbonyl)-3,4-tetrazolium]-bis(4-methoxy-6-nitro)benzenesulfonic acid hydrate and $N$-methyl dibenzopyrazine methyl sulfate) assay was performed to examine the effects of purslane on the viability of BMMs and RAW264.7 cells. Bone marrow-derived macrophages $\left(1 \times 10^{4}\right.$ cells/well $)$ were seeded in 96-well plates with various concentrations of purslane and incubated for $3 \mathrm{~d}$ in the presence of M-CSF (30 ng/ $\mathrm{mL})$. Subsequently, XTT solution $(50 \mu \mathrm{L})$ was added to each well and incubated for $4 \mathrm{~h}$. The plate was read at $450 \mathrm{~nm}$ with an ELISA reader (Molecular Devices, CA, U.S.A.). RAW264.7 cells were seeded in 48 -well plates at a density of $3 \times 10^{3}$ cells/ well and cultured for $4 \mathrm{~d}$ in the presence of various concentrations of purslane. After $4 \mathrm{~d}$, the cells were fixed with $3.7 \%$ formalin and stained with hematoxylin. Colonies with 50 cells or greater were counted.

Actin Ring Assay Bone marrow-derived macrophages were incubated with M-CSF (30 ng/mL) and RANKL (100 ng/ $\mathrm{mL}$ ) in the presence of dimethyl sulfoxide (DMSO, vehicle) or purslane $(0-50 \mu \mathrm{g} / \mathrm{mL})$ for $4 \mathrm{~d}$. The cells were fixed in $3.7 \%$ formalin for $20 \mathrm{~min}$ and permeabilized with $0.1 \%$ Triton X-100 for $15 \mathrm{~min}$. The cells were adapted in $0.25 \%$ bovine serum albumin (BSA) for $30 \mathrm{~min}$ followed by staining with phalloidin and DAPI solution (Life Technologies, Carlsbad, CA, U.S.A.).

Pit Formation Assay Bone marrow-derived cells $\left(1 \times 10^{7}\right.$ cells) and primary osteoblasts $\left(1 \times 10^{6}\right.$ cells) were seeded on collagen gel-coated culture dishes and cultured for $7 \mathrm{~d}$ in the presence of $10^{-8} \mathrm{M}$ 1,25-dihydroxyvitamin $\mathrm{D}_{3}$ (Sigma) and $10^{-6} \mathrm{M}$ prostaglandin $\mathrm{E}_{2}\left(\mathrm{PGE}_{2}\right)$ (Sigma). The co-cultured cells were detached by $0.1 \%$ collagenase treatment at $37^{\circ} \mathrm{C}$ for $10 \mathrm{~min}$ and were then replated on hydroxyapatite-coated plates (Corning, Corning, NY, U.S.A.). The cells were incubated on the plates with or without purslane. After $24 \mathrm{~h}$ the cells were removed and the total resorption pits were photographed and analyzed using the Image-Pro Plus software, version 4.0 (Media Cybernetics, Silver Spring, MD, U.S.A.).

Quantitative Real-Time Reverse Transcription Polymerase Chain Reaction (RT-PCR) Total RNA was isolated with QIAzol reagent (QIAGEN, Valencia, CA, U.S.A.) according to the manufacturer's instructions. To obtain cDNA, equal amounts of total RNA were reverse-transcribed into cDNA using SuperScript II Reverse Transcriptase (Invitrogen, San Diego, CA, U.S.A.). Real-time RT-PCR was performed in a $20 \mu \mathrm{L}$ reaction mixture containing $10 \mu \mathrm{L}$ of SYBR Green Premix (Bioneer Co., Daejeon, Korea), 10 pmol of forward primer, $10 \mathrm{pmol}$ of reverse primer, and $1 \mu \mathrm{g}$ of cDNA using 
a Exicycler ${ }^{\mathrm{TM}} 96$ Real-Time Quantitative Thermal Block (Bioneer Co.). The following primers were used to detect the genes of interest: $c$-Fos, forward 5'-GGT GAA GAC CGT GTC AGG AG-3' and reverse 5'-TAT TCC GTT CCC TTC GGA TT-3'; NFATc1, forward 5'-GAG TAC ACC TTC CAGCAC CTT-3' and reverse 5'-TATGATGTCGGGGAAAGA GA-3'; osteoclastassociated recepter (OSCAR), forward 5'-GGAATGGTCCT CATC TGC TT- $3^{\prime}$ and reverse $5^{\prime}$-GGA ATG GTCCTC ATC TG C TT-3'; TRAP, forward 5'-TCA TGGGTGGTGCTGCT-3' and reverse 5'-GCCCACAGCCACAAA TCT-3'; dendritic cellspecific transmembrane (DC-STAMP), forward 5'-GCAAGG AACCCAAGGAGTCG-3' and reverse 5'-CAGTTGGCC CAGAAA GAGGG-3'; OC-STAMP, forward 5'-TGGGCC TCCATA TGA CCTCGA GTA G-3' and reverse $5^{\prime}$-TCAAAG GCT TGT AAA TTGGAGGAGT-3'; integrin $\alpha v$, forward 5'TTG TTGCCGCCT TACGAGAA-3' and reverse 5'-GCAGAT GGCATA GCC ACA GG-3'; integrin $\beta 3$, forward 5'-TCTCCT GCG TCC GCT ACAAA-3' and 5'-CCC TTGGGACACTCA GGCTC-3'; ATP6v0d2, forward 5'-GACCCTGTGGCACTT TTT GT-3' and reverse 5'-GTG TTT GAGCTT GGGGAGAA3'; Cathepsin $K$, forward 5'-CACTGC TCTCTTCAGGGC TT-3' and reverse 5'-ACGGAGGCA TTGACTCTGAA-3'; and glyceraldehyde-3-phosphate dehydrogenase (GAPDH), forward 5'-TCA AGA AGG TGGTGA AGCAG-3' and reverse 5'-AGT GGG AGT TGC TGT TGA AGT-3'. The mouse GAPDH gene was used as internal control. The amplification parameters consisted of an initial denaturation step at $95^{\circ} \mathrm{C}$ for $5 \mathrm{~min}$ followed by 40 cycles of denaturation at $95^{\circ} \mathrm{C}$ for $1 \mathrm{~min}$, annealing at $60^{\circ} \mathrm{C}$ for $30 \mathrm{~s}$, and extension at $72^{\circ} \mathrm{C}$ for $1 \mathrm{~min}$. The specificity of the SYBR green assays was confirmed by melting-point analysis. Expression data were calculated from the cycle threshold $\left(C_{\mathrm{t}}\right)$ value using the $C_{\mathrm{t}}$.

Western Blot Analyses Whole-cell lysates were prepared using lysis buffer containing $50 \mathrm{~mm}$ Tris- $\mathrm{HCl}, 150 \mathrm{~mm} \mathrm{NaCl}$, $5 \mathrm{~mm}$ ethylenediaminetetraacetic acid (EDTA), 1\% Triton $\mathrm{X}-100,1 \mathrm{~mm}$ sodium fluoride, $1 \mathrm{~mm}$ sodium vanadate, $1 \%$ deoxycholate, and protease inhibitors. The cell suspension was centrifuged at $14000 \times \boldsymbol{g}$ for $20 \mathrm{~min}$ and the supernatant was used as the nuclear extract. Protein content was measured using a Bio-Rad colorimetric protein assay kit (Bio-Rad Laboratories Inc., Hercules, CA, U.S.A.). Equal amounts of protein $(20 \mu \mathrm{g})$ were run on $8-10 \%$ sodium dodecyl sulfatepolyacrylamide gel electrophoresis (SDS-PAGE) gels and transferred by electroblotting onto polyvinylidene difluoride membranes (Millipore, Bedford, MA, U.S.A.). The membranes were blocked with $5 \%$ nonfat milk in Tris-buffered saline contacting $0.1 \%$ Tween-20 (TBST) for $1 \mathrm{~h}$, before blotting with the primary antibodies for $2 \mathrm{~h}$ at room temperature. The membranes were washed in TBST, and incubated for $1 \mathrm{~h}$ with horseradish peroxidase (HRP)-conjugated sheep anti-mouse or donkey anti-rabbit immunoglobulin antibodies. Specific signals were detected using the Western chemiluminescent HRP substrate kit (Millipore).

Model of LPS-Induced Bone Loss and Treatment Male,
A

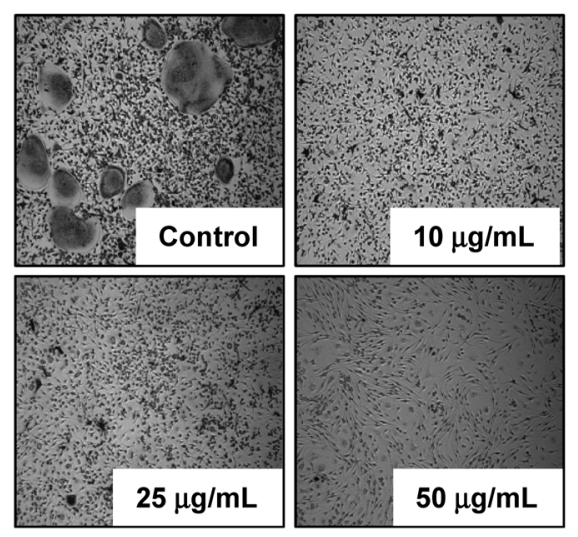

D

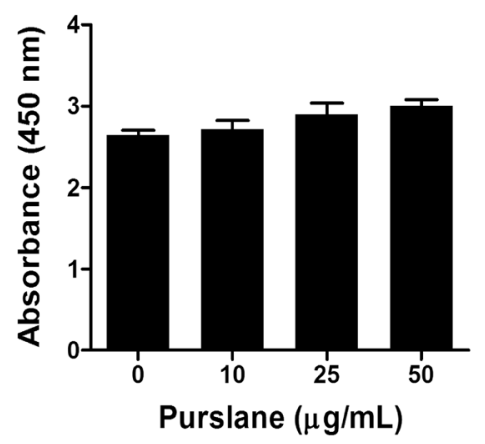

B

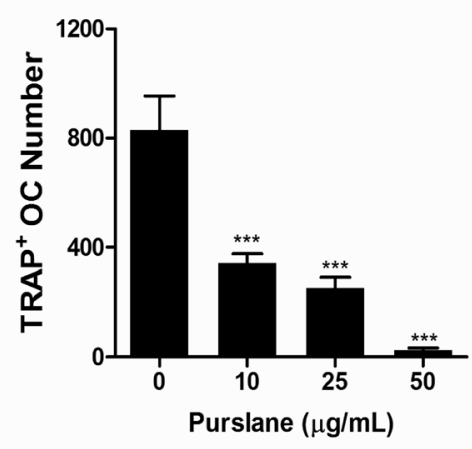

E

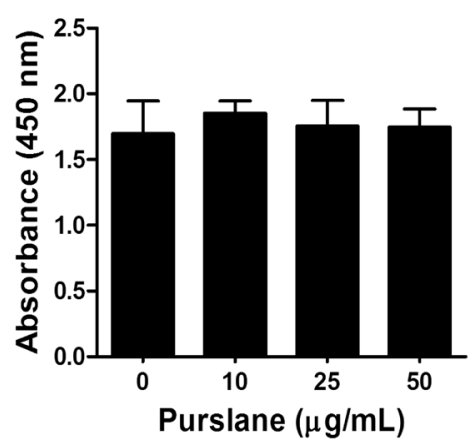

C

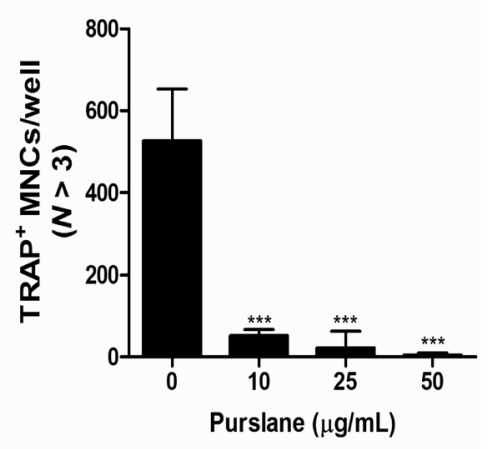

$\mathbf{F}$

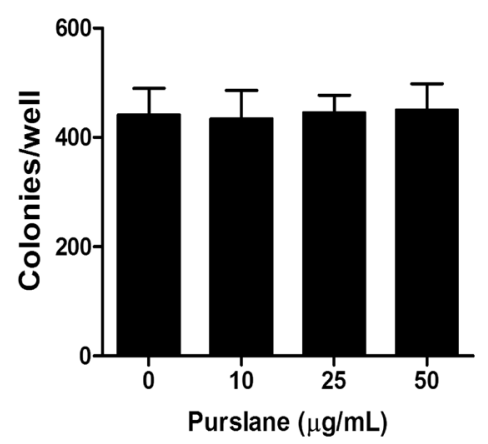

Fig. 1. Purslane Inhibits RANKL-Induced Osteoclast Differentiation in BMMs

BMMs were incubated in the presence of M-CSF $(30 \mathrm{ng} / \mathrm{mL})$ and RANKL $(100 \mathrm{ng} / \mathrm{mL})$ with control (DMSO) or purslane (10, 25, and 50 $\mu \mathrm{g} / \mathrm{mL})$ for $4 \mathrm{~d}$. (A) Representative microscopic pictures of TRAP staining. (B, C) The number of TRAP-positive osteoclasts or TRAP-positive MNCs having more than 3 nuclei was counted, respectively. (D, E) Cell viability was determined by XTT assay. BMMs were incubated in the presence of M-CSF ( $30 \mathrm{ng} / \mathrm{mL})$ with or without purslane for $3 \mathrm{~d}$ or (E) in the presence of M-CSF $(30 \mathrm{ng} / \mathrm{mL})$ and RANKL $(100 \mathrm{ng} / \mathrm{mL})$ with the indicated concentrations purslane for $4 \mathrm{~d}$. (F) RAW264.7 cells were seeded at 3000 cells/well in 48 -well plates and cultured with the indicated concentrations of purslane for $3 \mathrm{~d}$. The cells were fixed and stained with hematoxylin. Colonies with 50 cells were counted. $* * * p<0.001$ versus Control. 
5-week-old ICR mice were purchased from Damul Science (Daejeon, Korea). The mice were kept in a temperature $\left(22-24^{\circ} \mathrm{C}\right)$ and humidity $(55-60 \%)$ controlled environment with a $12 \mathrm{~h}$ light/dark cycle. All experiments were performed in accordance with the guidelines for animal experimentation of the Institute Committee of Wonkwang University. To study the effect of purslane on LPS-induced osteoclast formation in vivo, ICR mice were divided into 4 experimental groups comprised of 5 mice each: a phosphate-buffered saline (PBS)treated (Control), LPS-treated, purslane-treated, and LPS and purslane-treated group. Purslane $(250 \mathrm{mg} / \mathrm{kg})$ or PBS was administered orally $1 \mathrm{~d}$ before LPS injection $(5 \mathrm{mg} / \mathrm{kg}$ in $200 \mu \mathrm{L})$ and then every other day for $8 \mathrm{~d}$. LPS was injected intraperitoneally on days 1 and 4 .

Micro-Computed Tomography $(\mu \mathrm{CT})$ and Histological Analyses The mice were sacrificed $8 \mathrm{~d}$ after PBS, LPS, purslane, or LPS and purslane treatment, and the left femurs were analyzed by high-resolution $\mu \mathrm{CT}$. The femur metaphysic regions were scanned using a high-resolution $\mu \mathrm{CT}$ (NFRPolaris-S160; Nanofocus Ray, Iksan, Korea) with a source voltage of $60 \mathrm{kVp}$, current of $114 \mu \mathrm{A}$, and $7-\mu \mathrm{m}$ isotropic resolution. Femur scans were performed from the growth plate proximally to $2 \mathrm{~mm}$, with 350 sections per scan. Bone histomorphometric analyses were performed with $\mu \mathrm{CT}$ data using INFINITT-Xelis software (INFINITT Healthcare, Seoul, Korea). The structural parameters measured were trabecular bone volume/total volume (BV/TV, \%), trabecular thickness (Tb.Th, $\mu \mathrm{m}$ ), trabecular separation (Tb.Sp, $\mu \mathrm{m}$ ) and trabecular number (Tb.N, 1/mm). The right femurs were fixed in $4 \%$ paraformaldehyde (Sigma) for $1 \mathrm{~d}$, decalcified for 3 weeks in $12 \%$ EDTA, and embedded in paraffin. Sections $(5 \mathrm{~mm}$ thick) were prepared using a Leica microtome RM2145 (Leica Microsystems, Bannockburn, IL, U.S.A.). For histologic examination, sections were stained with hematoxylin and eosin (H\&E), and a separate section was stained with TRAP to identify osteoclasts on the bone surface.

Statistical Analyses Experiments were conducted separately at least 3 times and all data are presented as the mean \pm standard deviation (S.D.). All statistical analyses were performed using SPSS software (Korean version 14.0). The ANOVA test followed by the Tukey post-hoc test was used to compare the parameters among 3 groups. A $p<0.05$ was con-
A
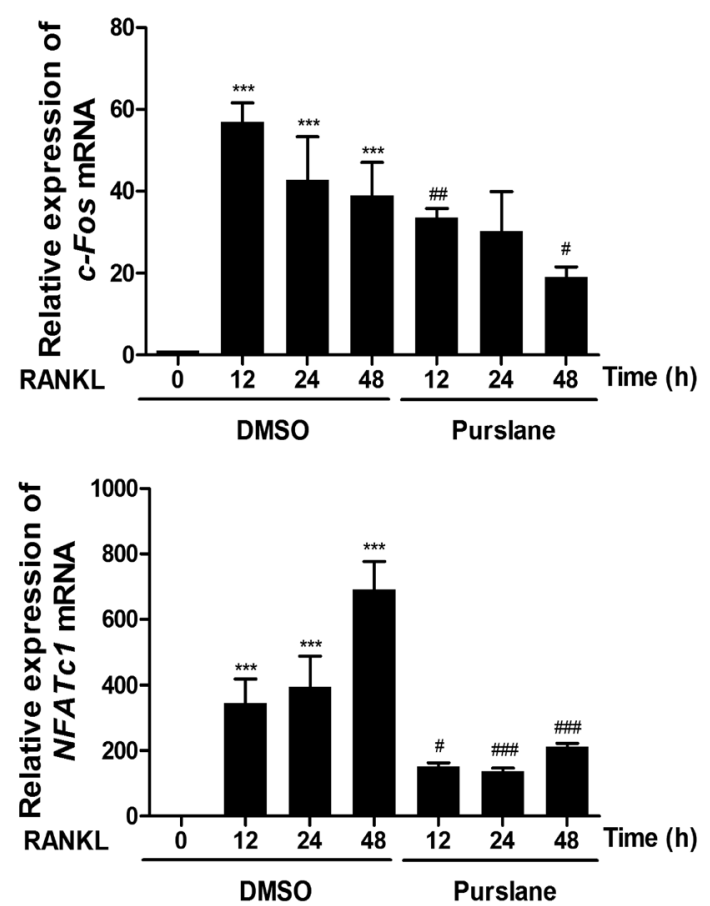

B

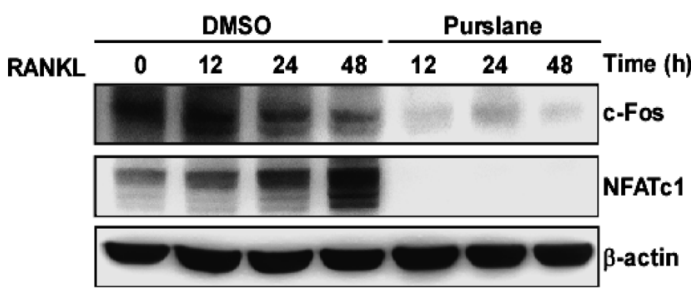

C

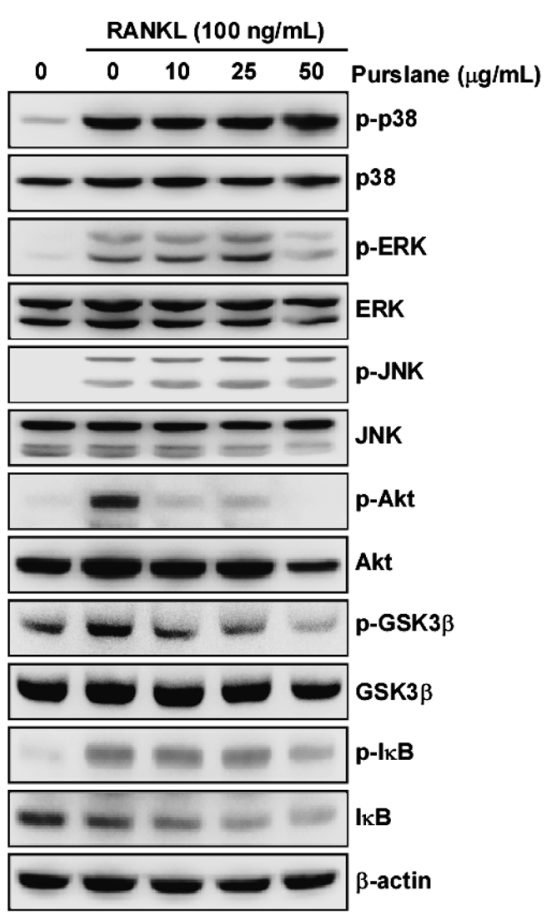

D

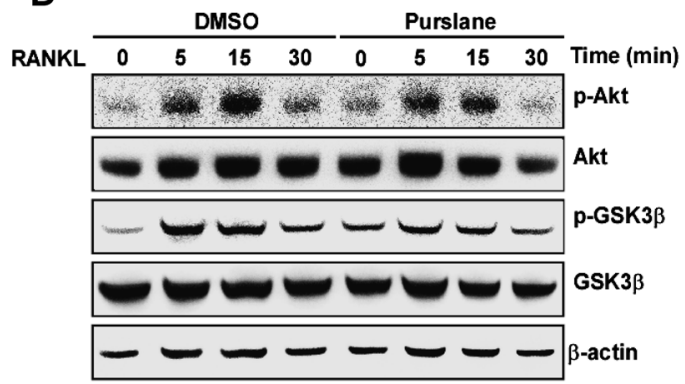

Fig. 2. Purslane Inhibited RANKL-Induced Activation of the Akt/GSK3 $\beta$-c-Fos-NFATc1 Signaling Pathway

(A, B) BMMs were pretreated with control (DMSO) or purslane $(50 \mu \mathrm{g} / \mathrm{mL})$ in the presence of M-CSF $(30 \mathrm{ng} / \mathrm{mL})$ for $1 \mathrm{~h}$ followed by stimulation with RANKL (100 ng/ $\mathrm{mL}$ ) for the indicated time period. (A) The mRNA expression of c-Fos and NFATc1 was analyzed by real-time RT-PCR. ${ }^{* * *} p<0.001$ versus Control; ${ }^{\#} p<0.05$, ${ }^{\#} p<0.01$, $\#$ \#\# $<0.001$ versus control at the indicated time. (B) Western blot analysis was performed with c-Fos and NFATc1 antibodies. (C, D) BMMs were pretreated with or without purslane $(50 \mu \mathrm{g} / \mathrm{mL})$ for $1 \mathrm{~h}$ before RANKL $(100 \mathrm{ng} / \mathrm{mL})$ stimulation at the indicated dose or time. Cell lysates were analyzed by Western blot analysis with the indicated antibodies. 
sidered statistically significant.

\section{RESULTS}

Purslane Inhibits RANKL-Induced Osteoclast Differentiation The effect of purslane on RANKL-induced osteoclastogenesis was evaluated in BMMs in the presence of M-CSF. Pre-treatment with purslane before RANKL treatment strongly prevented the RANKL-induced formation of TRAP-positive multinucleated cells in a dose-dependent manner (Fig. 1A). The inhibitory effect of purslane on osteoclast differentiation was confirmed by counting the number of TRAP-positive osteoclasts (Fig. 1B) or TRAP-positive multinucleated osteoclasts $(N<3)$ (Fig. 1C). Since the anti-osteoclastogenic activity of purslane could be due to its cytotoxicity in BMMs, we investigated such potential effect in BMMs. As shown in Figs. 1D-F, purslane did not exert cytotoxic effects at the same doses that effectively inhibited osteoclast differentiation, indicating that the inhibitory effect of purslane on osteoclast differentiation was not due to cellular toxicity or cell proliferation.

Purslane Inhibits RANKL-Induced Expression of c-Fos and NFATc1 To address the inhibitory mechanism of purslane on RANKL-induced osteoclastogenesis, we explored the effect of purslane on the expression of c-Fos and NFATc1, key transcription factors for osteoclastogenesis. Stimulation of BMMs with RANKL increased c-Fos mRNA with protein levels reaching a peak after $12 \mathrm{~h}$, which was followed by NFATc1 induction. However, the RANKL-induced expression of c-Fos and NFATc1 was strongly blocked by purslane (Figs. 2A, B).

Purslane Inhibits RANKL-Induced Phosphorylation of Akt and GSK3 $\beta$ Mitogen-activated protein kinase, Akt, GSK $3 \beta$, and the transcription factor NF- $\kappa \mathrm{B}$ signaling pathways are implicated in RANKL-induced c-Fos and NFATc1 expression. To elucidate the molecular mechanisms underlying the inhibitory effect of purslane on c-Fos and NFATc1 expres-

A

Purslane $(\mu \mathrm{g} / \mathrm{mL})$

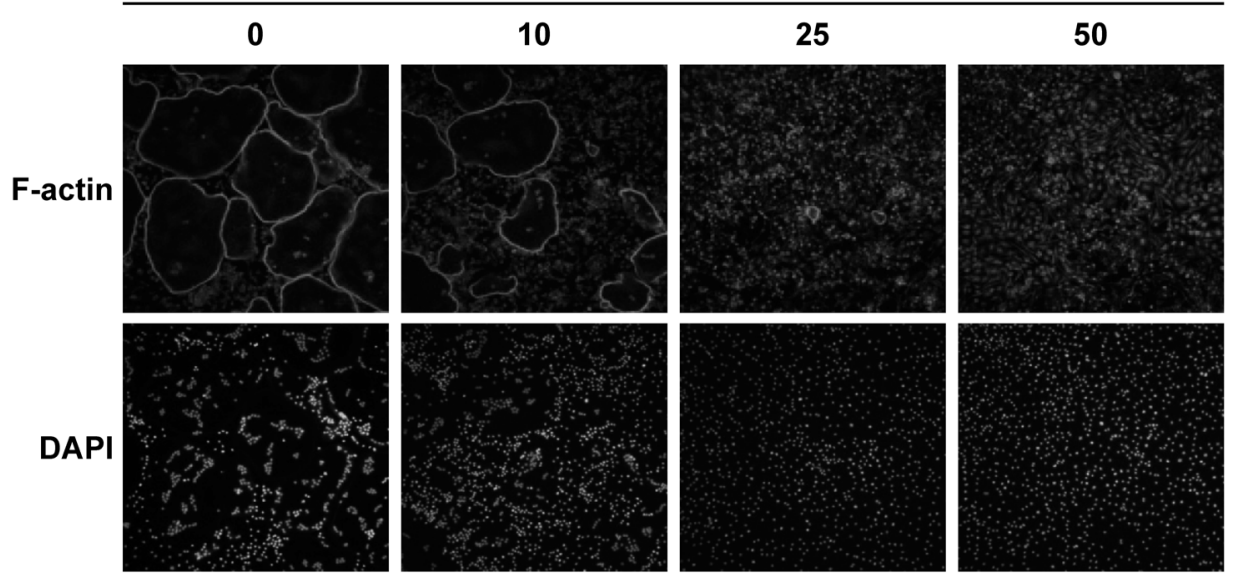

B
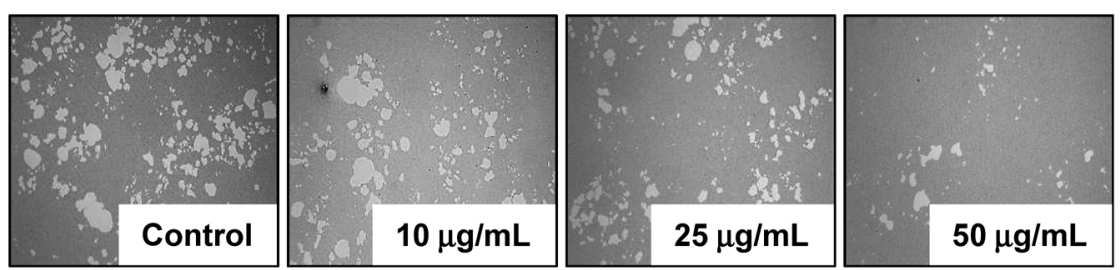

C
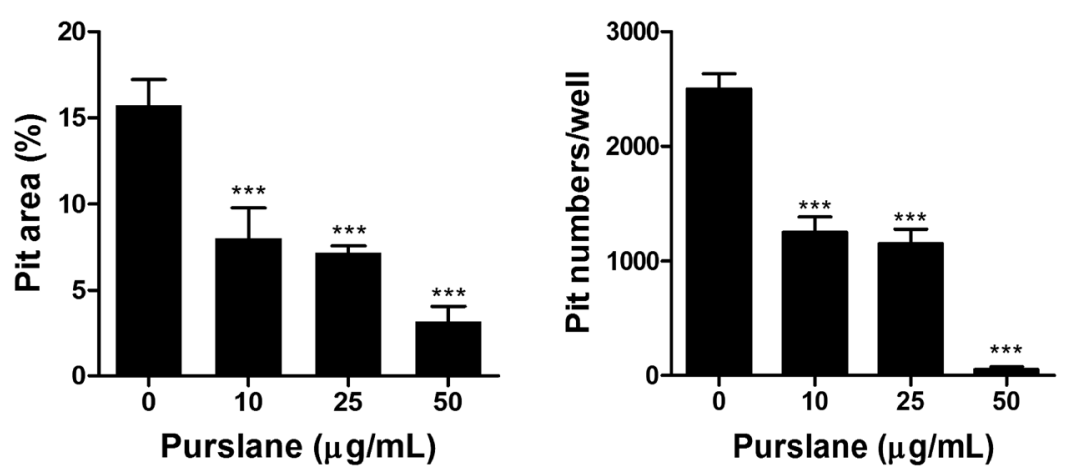

Fig. 3. Purslane Decreases Actin Ring Formation and Bone-Resorbing Activity of Mature Osteoclasts

(A) BMMs were cultured for $4 \mathrm{~d}$ in the presence of M-CSF $(30 \mathrm{ng} / \mathrm{mL})$ and RANKL $(100 \mathrm{ng} / \mathrm{mL})$ with control (DMSO) or various concentrations of purslane. Cells were fixed with 3.7\% formalin in PBS, permeabilized with $0.1 \%$ Triton X-100 in PBS, and stained with phalloidin and DAPI. (B) Mature osteoclasts were seeded on hydroxyapatite-coated plates for $24 \mathrm{~h}$ with the indicated concentrations of purslane. Cells attached to the plates were removed and photographed under a light microscope. (C) The relative ratio and number of pit areas were quantified using Image J software (U.S. National Institutes of Health, Bethesda, MD, U.S.A.). *** $p<0.001$ versus Control. 

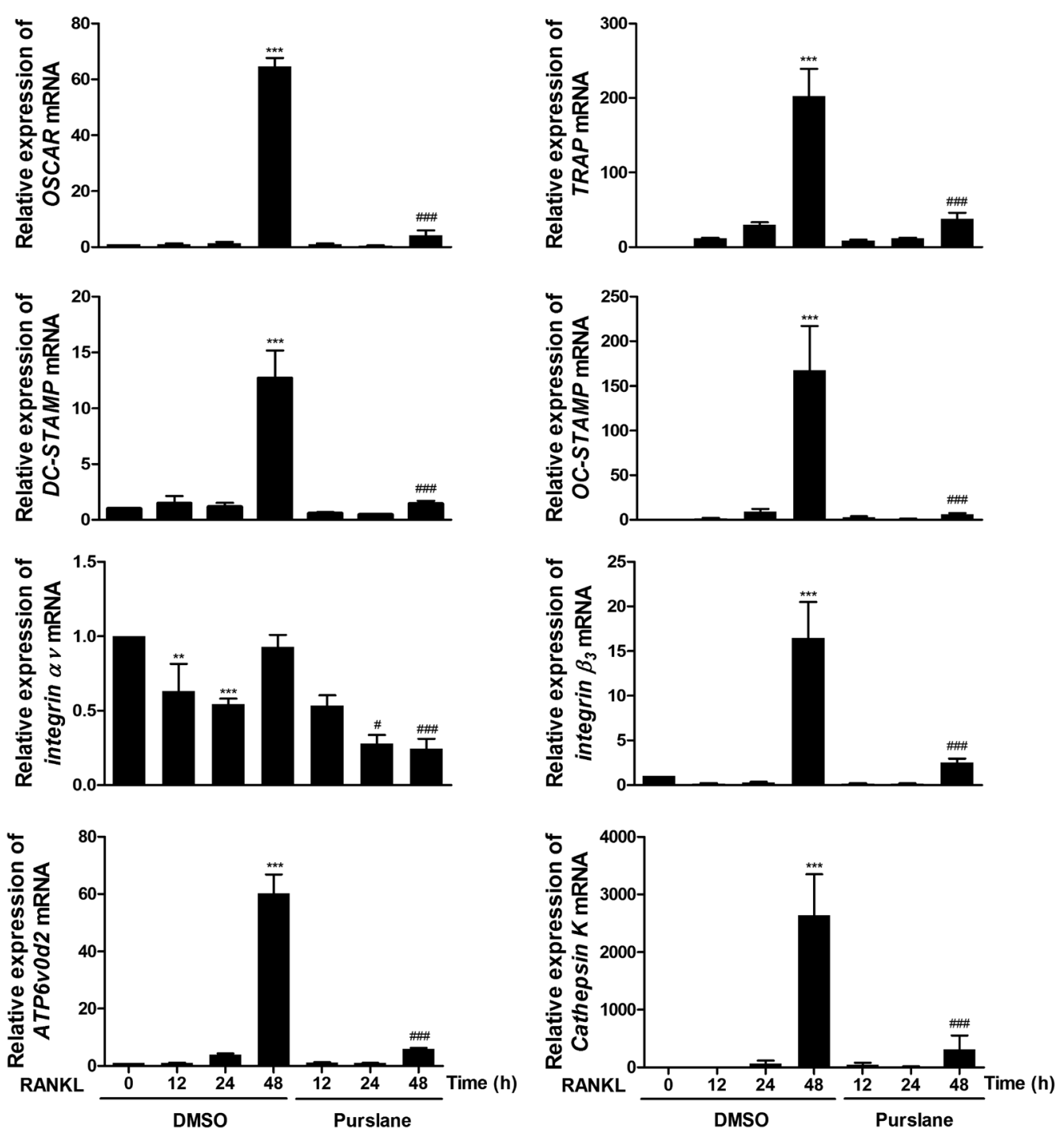

Fig. 4. Effect of Purslane on RANKL-Induced mRNA Expression of Osteoclast-Specific Genes

BMMs were treated with control (DMSO) or purslane $(50 \mu \mathrm{g} / \mathrm{mL})$ for $1 \mathrm{~h}$ followed by the addition of RANKL (100 ng/mL). The mRNA expression levels of osteoclastspecific genes were analyzed by real-time RT-PCR. ${ }^{* *} p<0.01,{ }^{* * *} p<0.001$ versus Control; ${ }^{\#} p<0.05$, ${ }^{\# \#} p<0.001$ versus Control at the indicated time.

sion, we explored whether purslane affects MAP kinase, Akt, GSK $3 \beta$, and NF- $\kappa$ B activation in response to RANKL. Stimulation of BMMs with RANKL rapidly activated MAP kinase, Akt, and GSK $3 \beta$ as well as the NF- $\kappa$ B signaling pathway (I $\kappa \mathrm{B}$ phosphorylation and degradation). We found that RANKLmediated induction of phosphorylation of p38, ERK, JNK, and $\mathrm{I} \kappa \mathrm{B}$ and degradation of $\mathrm{I} \kappa \mathrm{B}$ were not affected by purslane at different doses (Fig. 2C). In contrast, the phosphorylation of Akt and GSK3 $\beta$ was suppressed by purslane in a dose or time-dependent manner (Figs. 2C, D).

Purslane Inhibits Actin Ring Formation and BoneResorbing Activity of Mature Osteoclasts The actin ring is a distinctive cytoskeletal structure in mature osteoclasts and its formation is essential for osteoclastic bone resorption. Osteoclasts formed a ring-like F-actin structure, the actin ring, at the cell periphery. Treatment of osteoclasts with purslane resulted in disruption of the actin ring structure in a dose-dependent manner (Fig. 3A). Next, we investigated the effect of purslane on osteoclastic bone resorption. When mature osteoclasts were cultured for $24 \mathrm{~h}$ on hydroxyapatite-coated plates, an area of numerous resorbed pits by osteoclasts was formed in the vehicle-treated control. Purslane decreased the total resorbed area or number of resorbed pits in a dose-dependent manner (Figs. 3B, C).

Purslane Inhibits RANKL-Induced mRNA Expression of Osteoclast-Specific Genes To further confirm the inhibitory effect of purslane on osteoclast differentiation and function, the osteoclast gene expression profile was investigated by real-time RT-PCR analysis. As shown in Fig. 4, the expression of osteoclast-specific genes was gradually induced during osteoclastogenesis. Purslane downregulated the expression of $O S C A R$ and TRAP, which are genes specifically related to osteoclast differentiation. Moreover, purslane suppressed $D C$-STAMP, OC-STAMP, integrin $\alpha v$ and $\beta 3$, and ATP6v0d 2 expression, which are known to affect cell-cell interactions such as migration or fusion. Expression of cathepsin $K$, which is related to bone-resorbing activity, was also significantly inhibited.

Purslane Prevents Bone Destruction Induced by LPS in Vivo To address the effect of purslane in vivo, the inflammatory, LPS-induced osteolysis mouse model was chosen as previously described by our group. Mice were injected 
A
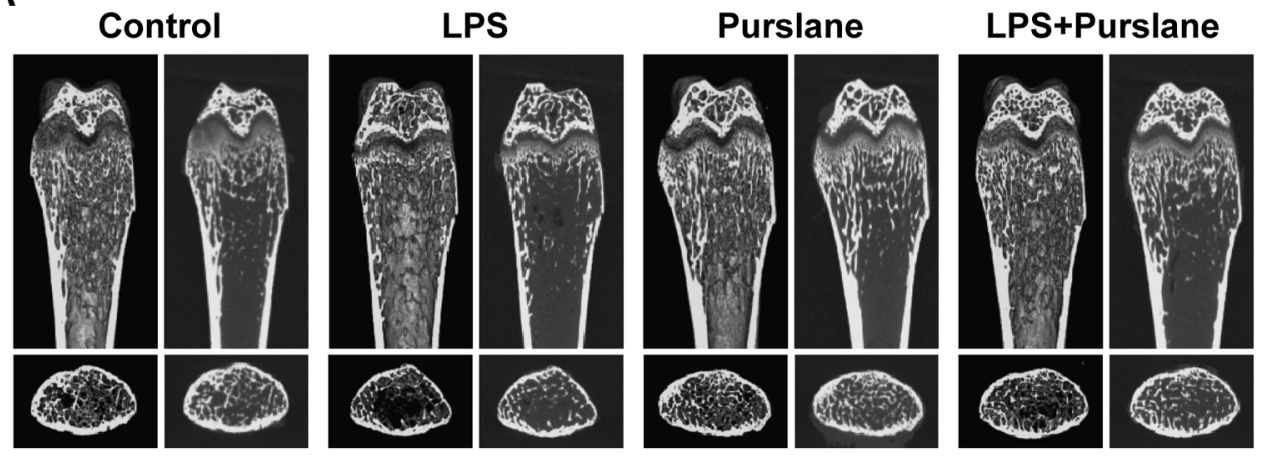

B
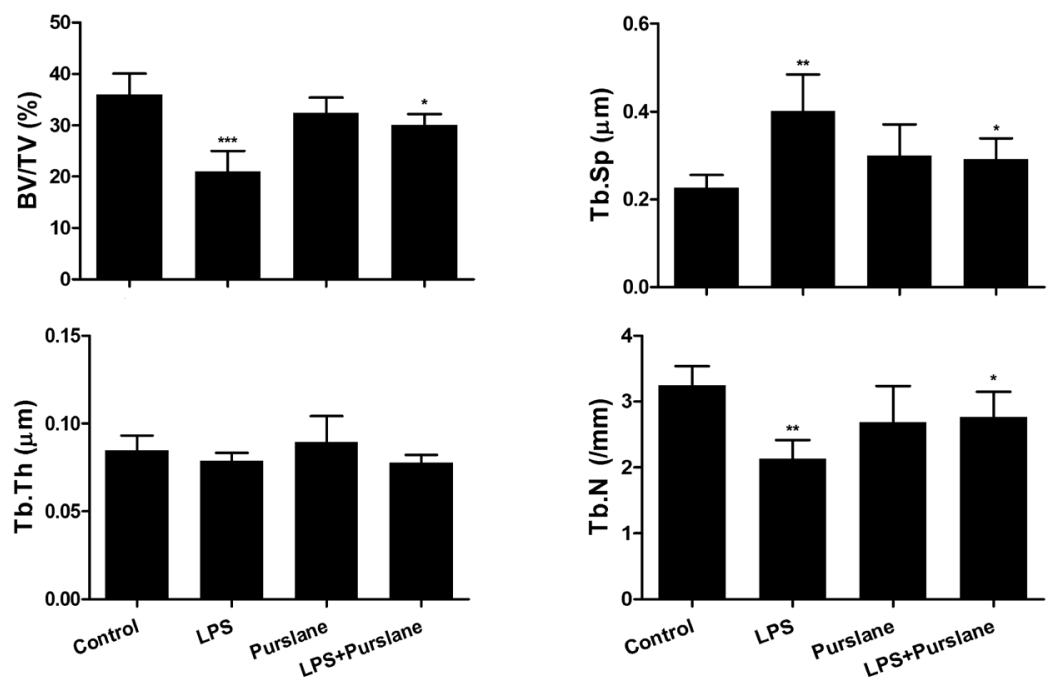

\section{Control}

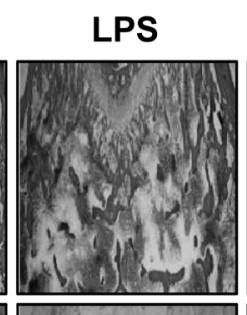

Purslane
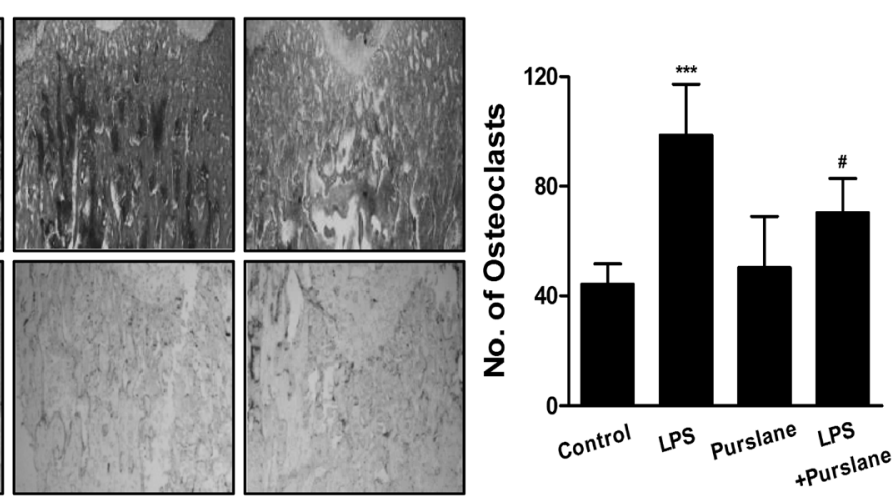

Fig. 5. Purslane Prevents LPS-Induced Bone Loss by Inhibiting Osteoclast Activity

(A) Mice were euthanized $8 \mathrm{~d}$ after the first LPS injection. Radiographs of longitudinal and transverse sections of a 2- or 3-dimensional visualization of the proximal femur were obtained with a $\mu \mathrm{CT}$ apparatus. (B) The trabecular bone volume/total volume (BV/TV), trabecular separation (Tb.Sp), trabecular thickness (Tb.Th), and trabecular number ( $\mathrm{Tb} . \mathrm{N})$ of the femurs were determined using the $\mu \mathrm{CT}$ data and INFINITT-Xelis software. (C) Dissected femora were fixed, decalcified, embedded, and sectioned. Sections were stained with H\&E and TRAP (left). The number of osteoclasts per filed of tissue was analyzed using the histomorphometric results (right). ${ }_{* *}^{*} p<0.01,{ }^{* * *} p<0.001$ versus Control group; ${ }^{*} p<0.05$ versus LPS group.

LPS intraperitoneally with or without purslane. No fatalities were recorded after LPS and purslane administration and the animals retained normal activity throughout the duration of the experiment. In the $\mu \mathrm{CT}$ analyses, a 2- or 3-dimensional visualization of the femoral area revealed a massive loss of trabecular bone following LPS treatment. On the other hand, LPS-induced bone loss was clearly reduced in the femurs of purslane-treated, LPS-injected mice (Fig. 5A). Morphometric analyses of the femurs from LPS-treated mice revealed pronounced reductions in the BV/TV, Tb.Sp, and Tb.N (Fig. 5B). We observed that the reduction of BV/TV, Tb.Sp, and Tb.N following LPS injection was recovered in the purslane-treated, LPS-induced mice (Fig. 5B). Histological examination confirmed the protective effects of purslane on LPS-induced bone loss. As shown in Fig. 5C, LPS injection led to bone erosion and increased numbers of TRAP-positive osteoclasts. However, bone erosion was rescued in femur tissue sections from purslane-treated mice, which was consistent with decreased TRAP-positive osteoclasts. Taken together, our data indicate that purslane prevented LPS-induced bone loss in vivo. 


\section{DISCUSSION}

An imbalance caused by an increased number or overactivation of osteoclasts can lead to impaired bone structure and low bone mass, which are common characteristics in patients with bone diseases. Therefore, a method to pharmaceutically inhibit osteoclast differentiation and function is one of the therapeutic strategies for preventing and/or treating bone diseases. In this study, we have verified for the first time that purslane inhibited osteoclast differentiation and bone resorption in vitro and in vivo, suggesting an additional protective effect of purslane on osteoclast-related diseases. In addition, we revealed that the molecular mechanisms of purslane on osteoclasts are through suppression of the Akt/GSK3 $\beta$ signaling pathway.

In osteoclasts, the Akt signaling cascade is a critical downstream pathway of 3 osteoclast surface receptors including c-fms, integrin $\alpha v \beta 3$, and RANK. ${ }^{17,18)}$ Akt has been known to play a critical role in the survival of osteoclasts rather than in osteoclast differentiation through the phosphoinositide 3-kinase (PI3K) signaling pathway. ${ }^{19,20)}$ However, a recent study showed the importance of the Akt-NFATc1 signaling axis in osteoclast differentiation. Inhibition of Akt phosphorylation by LY294002 resulted in the inhibition of osteoclast differentiation via regulation of RANKL-induced activation of NFATc1. ${ }^{18)}$ In addition, Akt induced osteoclastogenesis through the GSK3 $\beta /$ NFATc1 signaling cascade and silencing of Akt by small interfering RNA prevented RANKL-induced osteoclastogenesis. ${ }^{18)}$ Consistent with these studies, our data suggest that purslane inhibited Akt and GSK $3 \beta$ phosphorylation and thus suppressed the RANKL-induced NFATc1 expression, which are critical for osteoclast differentiation. Interestingly, in the process of elucidating the effect of purslane on the MAP kinase and NF- $\kappa$ B pathway, no significant inhibitory impact was observed.

The anti-osteoclastogenic action of purslane we observed could be due to its potential to inhibit the Akt/GSK3 $\beta$ signaling pathway that consequently down-regulates the expression and/or activity of c-Fos and NFATc1. In particular, NFATc1, which is mainly regulated by c-Fos during osteoclastogenesis, plays a role as the most distal transcription factor required for regulating the expression of osteoclast-specific genes including TRAP, OSCAR, DC-STAMP, OC-STAMP, integrin $\alpha v \beta 3, A T P 6 v 0 d 2$, and cathepsin K. TRAP and OSCAR are recognized as a marker of osteoclast differentiation and exhibit bone resorptive activity in lysosomes. ${ }^{21,22)}$ OC-STAMP, DC-STAMP, ATP6v0d2 and cathepsin $\mathrm{K}$ are well-known molecules for fusion and bone resorptive activity. ${ }^{23-25)}$ Integrin $\alpha v \beta 3$ is also required for the maintenance of the osteoclast actin cytoskeleton and the control of bone resorption. ${ }^{26)}$ Our data suggest that purslane significantly inhibited the RANKLinduced expression of c-Fos and NFATcl. Additionally, we found that purslane dampened cellular induction of TRAP, OSCAR, DC-STAMP, OC-STAMP, integrin $\alpha v \beta 3, A T P 6 v 0 d 2$, and cathepsin $K$ by RANKL. Furthermore, our data also suggest that purslane decreased bone resorption activity of mature osteoclasts, which was accompanied by a rapid disruption of the actin ring structure in mature osteoclasts via the regulation of these osteoclast-specific genes.

Consistent with the in vitro study, administration of purslane protected against bone loss induced by LPS, a cell component of Gram-negative bacteria. Since injection of LPS rapidly induces trabecular bone loss through stimulation of osteoclast differentiation and function, the protective effect of purslane on bone loss is mainly due to the suppression of osteoclast differentiation and function. However, we cannot exclude the possibility that purslane might affect osteoblastic bone formation. Further studies on the influence of purslane on osteoblasts might provide more insight into its inhibitory effect on osteoclastogenesis. Given the crucial role of excessive osteoclast activity in pathological bone destruction, our findings strongly suggest that purslane may be useful in preventing or treating various destructive bone diseases.

To our knowledge, this is the first report of the antiosteoclastogenic activity of purslane and its mode of action. Purslane inhibits osteoclast differentiation and function by inhibiting the Akt/GSK3 $\beta$-c-Fos-NFATc1 signaling pathway in osteoclast precursors, and by disrupting the actin ring in mature osteoclasts, respectively. Furthermore, our in vivo results further verified the bone protective role of purslane in the LPS-induced osteolysis model. These findings suggest that purslane may be useful in preventing or treating bone diseases associated with excessive bone loss.

Acknowledgments This study was supported by a Grant from the Wonkwang University in 2013 and the Korean Health Technology R\&D Project, Ministry of Health \& Welfare, Republic of Korea (HI11C1602).

Conflict of Interest The authors declare no conflict of interest.

\section{REFERENCES}

1) Boyle WJ, Simonet WS, Lacey DL. Osteoclast differentiation and activation. Nature, 423, 337-342 (2003).

2) Harada S, Rodan GA. Control of osteoblast function and regulation of bone mass. Nature, 423, 349-355 (2003).

3) Lacey DL, Timms E, Tan HL, Kelley MJ, Dunstan CR, Burgess T, Elliott R, Colombero A, Elliott G, Scully S, Hsu H, Sullivan J, Hawkins N, Davy E, Capparelli C, Eli A, Qian YX, Kaufman S, Sarosi I, Shalhoub V, Senaldi G, Guo J, Delaney J, Boyle WJ. Osteoprotegerin ligand is a cytokine that regulates osteoclast differentiation and activation. Cell, 93, 165-176 (1998).

4) Jurdic P, Saltel F, Chabadel A, Destaing O. Podosome and sealing zone: specificity of the osteoclast model. Eur. J. Cell Biol., 85 195-202 (2006).

5) Kong YY, Yoshida H, Sarosi I, Tan HL, Timms E, Capparelli C, Morony S, Oliveira-dos-Santos AJ, Van G, Itie A, Khoo W, Wakeham A, Dunstan CR, Lacey DL, Mak TW, Boyle WJ, Penninger JM. OPGL is a key regulator of osteoclastogenesis, lymphocyte development and lymph-node organogenesis. Nature, 397, 315-323 (1999).

6) Takayanagi H. Osteoimmunology: shared mechanisms and crosstalk between the immune and bone systems. Nat. Rev. Immunol., 7 292-304 (2007).

7) Grigoriadis AE, Wang ZQ, Cecchini MG, Hofstetter W, Felix R, Fleisch HA, Wagner EF. c-Fos: a key regulator of osteoclast-macrophage lineage determination and bone remodeling. Science, 266, 443-448 (1994).

8) Matsuo K, Galson DL, Zhao C, Peng L, Laplace C, Wang KZ, Bachler MA, Amano H, Aburatani H, Ishikawa H, Wagner EF. Nuclear factor of activated T-cells (NFAT) rescues osteoclastogenesis in precursors lacking c-Fos. J. Biol. Chem., 279, 26475-26480 
(2004).

9) Takayanagi H, Kim S, Koga T, Nishina H, Isshiki M, Yoshida H, Saiura A, Isobe M, Yokochi T, Inoue J, Wagner EF, Mak TW, Kodama T, Taniguchi T. Induction and activation of the transcription factor NFATc1 (NFAT2) integrate RANKL signaling in terminal differentiation of osteoclasts. Dev. Cell, 3, 889-901 (2002).

10) Yamashita T, Yao Z, Li F, Zhang Q, Badell IR, Schwarz EM, Takeshita S, Wagner EF, Noda M, Matsuo K, Xing L, Boyce BF. NF-kappaB p50 and p52 regulate receptor activator of NF-kappaB ligand (RANKL) and tumor necrosis factor-induced osteoclast precursor differentiation by activating c-Fos and NFATc1. J. Biol. Chem., 282, 18245-18253 (2007).

11) Uddin MK, Juraimi AS, Hossain MS, Nahar MA, Ali ME, Rahman MM. Purslane weed (Portulaca oleracea): a prospective plant source of nutrition, omega-3 fatty acid, and antioxidant attributes. ScientificWorldJournal, 2014, 951019 (2014).

12) Liu L, Howe P, Zhou YF, Xu ZQ, Hocart C, Zhang R. Fatty acids and beta-carotene in australian purslane (Portulaca oleracea) varieties. J. Chromatogr. A, 893, 207-213 (2000).

13) Xiang L, Xing D, Wang W, Wang R, Ding Y, Du L. Alkaloids from Portulaca oleracea L. Phytochemistry, 66, 2595-2601 (2005).

14) Chan K, Islam MW, Kamil M, Radhakrishnan R, Zakaria MN, Habibullah M, Attas A. The analgesic and anti-inflammatory effects of Portulaca oleracea L. ssp. Sativa (Haw.) Celak. J. Ethnopharmacol., 73, 445-451 (2000).

15) Silva R, Carvalho IS. In vitro antioxidant activity, phenolic compounds and protective effect against DNA damage provided by leaves, stems and flowers of Portulaca oleracea (PursLane). Nat. Prod. Commun., 9, 45-50 (2014).

16) Rashed AN, Afifi FU, Disi AM. Simple evaluation of the wound healing activity of a crude extract of Portulaca oleracea L. (growing in Jordan) in Mus musculus JVI-1. J. Ethnopharmacol., 88, 131-136 (2003).

17) Cao H, Zhu K, Qiu L, Li S, Niu H, Hao M, Yang S, Zhao Z, Lai Y, Anderson JL, Fan J, Im HJ, Chen D, Roodman GD, Xiao G. Critical role of AKT protein in myeloma-induced osteoclast formation and osteolysis. J. Biol. Chem., 288, 30399-30410 (2013).

18) Moon JB, Kim JH, Kim K, Youn BU, Ko A, Lee SY, Kim N. Akt induces osteoclast differentiation through regulating the GSK3beta/ NFATc1 signaling cascade. J. Immunol., 188, 163-169 (2012).

19) Gingery A, Bradley E, Shaw A, Oursler MJ. Phosphatidylinositol 3-kinase coordinately activates the MEK/ERK and AKT/NF-kappaB pathways to maintain osteoclast survival. J. Cell. Biochem., 89, 165-179 (2003).

20) Wong BR, Besser D, Kim N, Arron JR, Vologodskaia M, Hanafusa $\mathrm{H}$, Choi Y. TRANCE, a TNF family member, activates Akt/PKB through a signaling complex involving TRAF6 and c-Src. Mol. Cell, 4, 1041-1049 (1999).

21) Angel NZ, Walsh N, Forwood MR, Ostrowski MC, Cassady AI, Hume DA. Transgenic mice overexpressing tartrate-resistant acid phosphatase exhibit an increased rate of bone turnover. J. Bone Miner. Res., 15, 103-110 (2000).

22) Nemeth K, Schoppet M, Al-Fakhri N, Helas S, Jessberger R, Hofbauer LC, Goettsch C. The role of osteoclast-associated receptor in osteoimmunology. J. Immunol., 186, 13-18 (2011).

23) Delaissé J-M, Andersen TL, Engsig MT, Henriksen K, Troen T, Blavier L. Matrix metalloproteinases (MMP) and cathepsin K contribute differently to osteoclastic activities. Microsc. Res. Tech., 61, 504-513 (2003)

24) Kim K, Lee SH, Ha Kim J, Choi Y, Kim N. NFATc1 induces osteoclast fusion via up-regulation of Atp6v0d2 and the dendritic cellspecific transmembrane protein (DC-STAMP). Mol. Endocrinol., 22, 176-185 (2008).

25) Yang M, Birnbaum MJ, MacKay CA, Mason-Savas A, Thompson $\mathrm{B}$, Odgren PR. Osteoclast stimulatory transmembrane protein (OCSTAMP), a novel protein induced by RANKL that promotes osteoclast differentiation. J. Cell. Physiol., 215, 497-505 (2008).

26) Nakamura I, Duong LT, Rodan SB, Rodan GA. Involvement of alpha(v)beta3 integrins in osteoclast function. J. Bone Miner. Metab., 25, 337-344 (2007). 